\title{
Expanding the comprehensive national neonatal screening programme in the United Arab Emirates from 1995 to 2011
}

\author{
H. Al Hosani, ${ }^{1}$ M. Salah, ${ }^{2}$ H.M. Osman, ${ }^{3}$ H.M. Farag, ${ }^{2}$ L. El-Assiouty, ${ }^{2}$ D. Saade and J. Hertecant $^{4}$
}

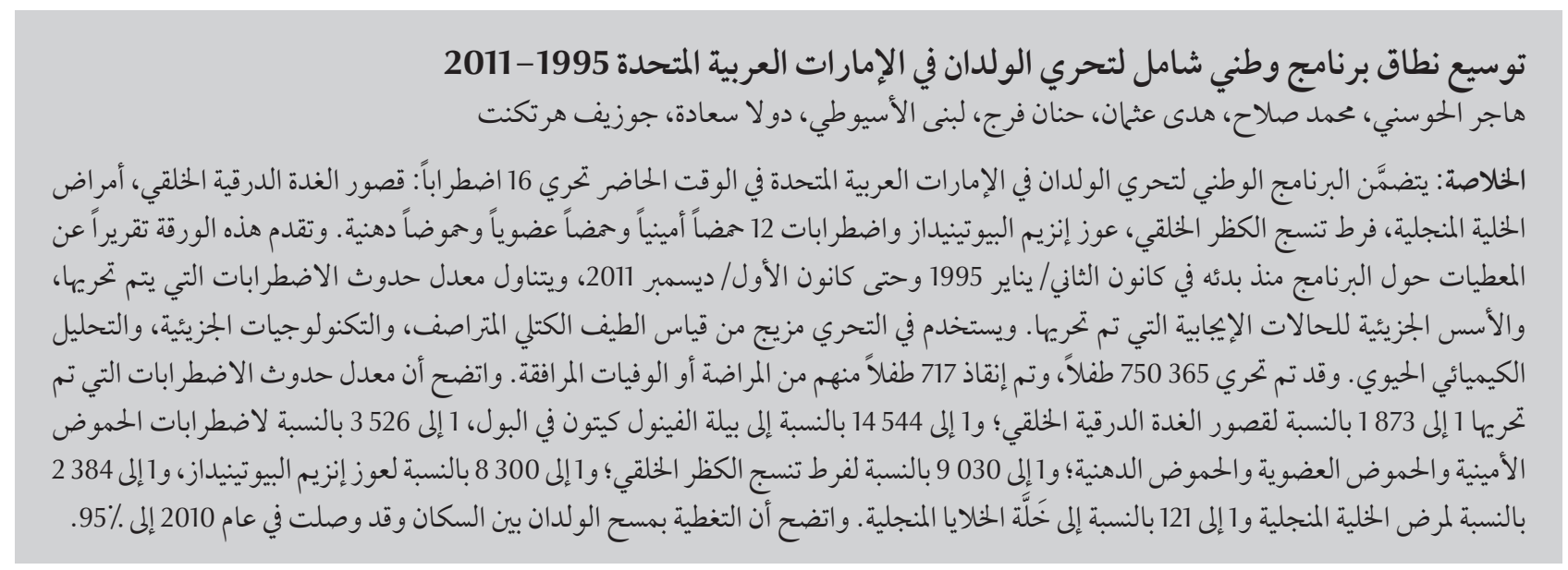

ABSTRACT The national neonatal screening programme in the United Arab Emirates currently includes 16 disorders: congenital hypothyroidism, sickle-cell diseases, congenital adrenal hyperplasia, biotinidase deficiency and 12 amino acid, organic acid and fatty acid disorders. This paper reports data since the programme started in January 1995 up to December 2011 on the incidence of screened disorders and the molecular basis of positive screened cases. Screening used a combination of tandem mass spectrometry, molecular technologies and biochemical analysis. A total of 750365 infants were screened and 717 babies saved from associated morbidity and/or mortality. The incidence of screened disorders were 1:1 873 for congenital hypothyroidism, 1:14 544 for phenylketonuria, 1:3526 for amino acid, organic acid and fatty acid disorders, 1:9 030 for classical congenital adrenal hyperplasia, 1:8 300 for biotinidase deficiency, 1:2 384 for sickle-cell disease and 1:121 for sickle-cell traits. Coverage of neonatal screening in the population reached 95\% in 2010.

\section{Extension du vaste programme national de dépistage néonatal aux Émirats arabes unis de 1995 à 2011}

RÉSUMÉ Le programme national de dépistage néonatal aux Émirats arabes unis couvre actuellement 16 maladies ou troubles : I'hyperthyroïdie congénitale, la drépanocytose, l'hyperplasie congénitale des surrénales, le déficit en biotinidase ainsi que 12 troubles des acides aminés, organiques et gras. L'article présente les données collectées, depuis le commencement du programme en janvier 1995 jusqu'en décembre 2011, sur l'incidence des troubles dépistés ainsi que la base moléculaire des cas positifs dépistés. La spectrométrie de masse en tandem, les technologies moléculaires et l'analyse biochimique ont été utilisées pour les besoins du dépistage. Au total, 750365 nourrissons ont été dépistés et la morbidité et/ou mortalité associée a pu être évitée pour 717 bébés. L'incidence des maladies ayant fait l'objet d'un dépistage était la suivante : 1 : 1873 pour l'hyperthyroïdie congénitale, 1 : 14544 pour la phénylcétonurie, $1: 3526$ pour les troubles des acides aminés, organiques et gras, $1: 9030$ pour la forme classique de I'hyperplasie congénitale des surrénales, $1: 8300$ pour le déficit en biotinidase, 1:2384 pour la drépanocytose et 1 : 121 pour les traits drépanocytaires. En 2010, la couverture de la population par le dépistage néonatal avait atteint 95 \%.

'Central Department of Maternal and Child Health, Ministry of Health, Abu Dhabi, United Arab Emirates (Correspondence to H. Al Hosani: hager@emirates.net.ae).

${ }^{2}$ Department of Genetics; ${ }^{3}$ National Screening Laboratory, National Screening Centre, Abu Dhabi, United Arab Emirates.

${ }^{4}$ Department of Paediatrics, Tawam Hospital, Abu Dhabi, United Arab Emirates.

Received: 09/02/12; accepted: 13/08/13 


\section{Introduction}

The concept of screening newborns no longer refers only to the screening tests themselves but includes all of the elements essential for every neonate to have access to a screening system that is optimal in terms of quality and performance [1].

The United Arab Emirates (UAE) has a universal neonatal screening programme which started in January 1995 with screening for phenylketonuria (PKU) followed by the introduction of congenital hypothyroidism (CH) screening in January 1998. A sickle-cell disease (SCD) screening was begun as a pilot study in January 2002 before expansion in March 2005 to the national level. Congenital adrenal hyperplasia $(\mathrm{CAH})$ screening also started as a pilot study in January 2005 before expansion to the national level in 2007. Screening for biotinidase deficiency was originally piloted in January 2010. Tandem mass spectrometry (MS/MS) technology expands the metabolic disorder screening panel and can reliably analyse approximately 50 metabolites in a single short-duration run of about 2 minutes and provide a comprehensive assessment from a single blood-spot specimen [2]. MS/MS was started in the UAE from March 2011 to screen for 12 amino acid, organic acid and fatty acid disorders.

The aim of the current study was to report data from the screening programme on the incidence of PKU, $\mathrm{CH}$, $\mathrm{CAH}, \mathrm{SCD} /$ trait, biotinidase deficiency, amino acid disorders, organic acid disorders and fatty acid disorders. It also aimed to identify the molecular basis of positive screened cases.

\section{Methods}

The sample forms were collected from the various maternal and child health centres throughout the UAE. The data from these forms and the results of the screening were statistically analysed in the computer section of the national screening programme. The screening protocol utilized a combination of MS/ MS, molecular technologies and biochemical analysis.

\section{Screening procedures}

In the UAE, every baby born in hospital (around 80000 babies with 99.9\% hospital deliveries in 2010) is given a specific neonatal screening form and an information leaflet. The mother is asked to take her baby to the nearest screening centre, usually the maternal and child health $(\mathrm{MCH})$ centre of that district. The baby attends the designated centre on the third day after birth ( $\geq 48$ hours) and blood is collected by heel-prick onto filter paper (S\&S 903) by technicians or trained nurses. For babies remaining in hospital for 3 days or more, blood is collected before departure. Quality assessment of laboratory results in the UAE is monitored by the schemes of the United Kingdom National External Quality Assessment Service and the United States Centers for Disease Control and Prevention. Any positive screening results are notified immediately to the central department of the $\mathrm{MCH}$ by the laboratory. The district $\mathrm{MCH}$ coordinator will be informed and will contact the parents.

For a baby screening positive for amino acid, organic acid and fatty acid oxidation disorders, $\mathrm{CH}, \mathrm{CAH}$ and biotinidase deficiencies, further blood and urine samples are tested to confirm the diagnosis. For a baby screening positive for SCD or trait, blood is taken from the baby and family members before the age of 2 months for diseased and 4 months for trait cases to confirm the diagnosis and deliver genetic counselling. Testing of the parents for all haemoglobinopathies, including thalassaemia, may help establish the correct diagnosis in some infants.

\section{Laboratory procedures}

For screening for PKU (before March 2011), CAH, CH and biotinidase deficiency the Delfia time-resolved fluorescence application (Wallac Oy) was used to determine levels of phenylalanine (PA), thyroid stimulating hormone (TSH), 17a-hydroxyprogesterone and biotinidase enzyme activity.

For detection of SCD the same filter papers were tested by high-performance liquid chromatography (Variant", BioRad).

Amino acid, organic acid and fatty acid oxidation disorders were detected by MS/MS (API 3200", HVD/Perkin Elmer). At the time of the study 12 disorders were included in the screening: PKU, maple syrup disease, citrullinaemia type I, argininosuccinic acidaemia, isovaleric acidaemia, 3-methylcrotonylCoA carboxylase deficiency, hydroxymethylglutaric aciduria, beta-ketothiolase deficiency, glutaric acidaemia type I, propionic acidaemia, methylmalonic acidaemia and medium-chain acylcoenzyme A dehydrogenase deficiency.

\section{Cut-offs}

The following cut-offs were used for TSH levels (normal $<10 \mathrm{mU} / \mathrm{L}$, borderline 10-25 mU/L, abnormal > 25 $\mathrm{mU} / \mathrm{L}$ ), PA levels (normal $<3 \mathrm{mg}$ / $\mathrm{dL}$, borderline 3-4 mg/dL, abnormal $>4 \mathrm{mg} / \mathrm{dL}$ ), 17OHP levels (normal < $30 \mathrm{nmol} / \mathrm{L}$, borderline $30-90 \mathrm{nmol} / \mathrm{L}$, abnormal $>90 \mathrm{nmol} / \mathrm{L}$ ) and biotinidase levels (normal $>30 \%$, borderline $10 \%-30 \%$, abnormal $<10 \%$ ).

\section{Molecular methods}

Molecular diagnosis is done for specimens that show putative positive results following initial analysis to determine the presence or absence of specific mutations. This provides critical genetic data to confirm diagnosis and genetic counselling. The tests are done for all neonates, but for this study we selected the results of the UAE national babies to determine the UAE genetic profile and we excluded from our study the genetic 
results of the other nationalities due to variable genetic profiles.

For PKU, the 13 exons and their exon-flanking intronic sequence of phenylalanine hydroxylase (PAH) gene were amplified by polymerase chain reaction (PCR) assay, and then singlestrand conformation polymorphism analysis for exons of the PAH gene and finally sequencing of gene exons was used to study uncharacterized PKU chromosomes [3]. The CAH confirmatory test was done by CAH strip assay covering 11 mutations in the CYP21A2 gene by reverse hybridization assay and a quantitative PCR approach to determine gene copy numbers [4]. For biotinidase deficiency, DNA sequence analysis was used to test for the presence of a mutation in all 4 exons of the biotinidase gene [5].

\section{Results}

\section{Uptake of screening}

Table 1 shows that the percentage uptake (coverage) of neonatal screening in the UAE increased from 50\% in 1998 to reach $95 \%$ in 2010, with a rapid increase in 2003.

\section{Congenital hypothyroidism}

A total of 513 infants who screened positive for $\mathrm{CH}$ were further investigated and followed up; these included 129 infants with persistent borderline $\mathrm{CH}$ (TSH $10-25 \mathrm{mU} / \mathrm{L}$ ) and 384 with abnormal results $(\mathrm{TSH}>25 \mathrm{mU} / \mathrm{L})$ (Table 2). Of the 129 borderline cases, $16(12.4 \%)$ were confirmed $\mathrm{CH}$, of whom 9 were defaulters. Of the infants with abnormal test results, 357 (92.9\%) were confirmed $\mathrm{CH}$. There were 60 transient cases that were normal on follow up. Among the transient cases, there were 14 cases with maternal history of autoimmune thyroid disease, 26 premature cases and 20 cases with unidentified causes. Of the 698629 babies screened from the start (1 January 1988 to 31 December 2011) 373 were confirmed $\mathrm{CH}$ cases, an incidence of $\mathrm{CH}$ among the screened neonates of 1:1 873.

\section{Phenylketonuria}

Table 3 shows 132 neonates who screened positive for PKU; these were 75 with persistent borderline PKU (phenylalanine $3-4 \mathrm{mg} / \mathrm{dL}$ ) and 57 abnormal babies (phenylalanine $>4 \mathrm{mg} / \mathrm{dL}$ )]. Of the 57 abnormal cases, 51 were confirmed as classic PKU (phenylalanine > $20 \mathrm{mg} / \mathrm{dL}$, normal tyrosine and normal biopterin) and 1 case as PKU due to biopterin defect. Of the borderline cases, $61(80.1 \%)$ were false positives. The incidence of PKU was 1:14 544.

\section{Molecular DNA testing}

A total of 7 different mutations ( 3 splicing mutations and 4 missense mutations) were detected by analysis of the PAH gene for 16 UAE national babies. The frequencies of the mutations were as follows: IVS2+5G>C (37.5\%), R261Q (18.8\%), IVS10-11G>A (12.5\%), IVS9-2A>G (12.5\%), R252Q (6.3\%), P281L (6.3\%) and L48S (6.3\%).

\section{Amino acid, organic acid and fatty acid disorders}

Table 4 shows that 16 cases of amino acid, organic acid or fatty acid disorders were detected by MS/MS from March 2011 until December 2011. There were 7 cases of hydroxymethylglutaric aciduria, 3 new cases of PKU (detected by MS/MS not by conventional methods) and 2 cases of glutaric acidaemia type I. The total incidence of these disorders was 1:353.

\section{Sickle cell haemoglobinopathies}

Table 5 shows that out of 5498 positive screening results for sickle-cell haemoglobinopathies 231 neonates were SCD (FS), 80 had non-sickle haemoglobinopathy, 4481 were sickle haemoglobinopathy carriers (FAS) and 592 were haemoglobinopathy D carriers (FAD). The data showed that the incidence of SCD was $0.04 \%$. It is noteworthy that 203 out of 231 SCD cases were confirmed as homozygous haemoglobin $(\mathrm{Hb}) \mathrm{S}$ and only 28 cases were confirmed as sickle/beta-thalassaemia. The incidence of sickle-cell traits were $0.83 \%$ (1:121). For the non-sickle haemoglobinopathies, the incidence was $0.11 \%$ for $\mathrm{HbD}$ trait and $0.02 \%$ for $\mathrm{HbC}$ trait.

\section{Congenital adrenal hyperplasia}

Table 6 shows that out of 42 positive CAH screening results 41 babies were

\begin{tabular}{lcc}
\hline $\begin{array}{l}\text { Table } 1 \text { Coverage of the neonatal screening programme in the United Arab } \\
\text { Emirates (1998 to 2010) }\end{array}$ & \% screened \\
\hline Year & No. of live births & 50 \\
1998 & 45044 & 61 \\
1999 & 49075 & 65 \\
2000 & 52070 & 67 \\
2001 & 53485 & 69 \\
2002 & 57083 & 89 \\
2003 & 60249 & 92 \\
2004 & 63610 & 94 \\
2005 & 66192 & 93 \\
2006 & 66967 & 94 \\
2007 & 67789 & 95 \\
2008 & 68779 & 95 \\
2009 & 76366 & 95 \\
2010 & 79464 & 95 \\
\hline
\end{tabular}

Data source: Department of Preventive Medicine, Ministry of Health, United Arab Emirates. 


\begin{tabular}{|c|c|c|c|c|c|}
\hline \multirow[t]{2}{*}{ Variable } & \multicolumn{2}{|c|}{$\begin{array}{c}\text { Borderline } \\
\text { (TSH 10-25 mU/L) }\end{array}$} & \multicolumn{2}{|c|}{$\begin{array}{c}\text { Abnormal } \\
(\mathrm{TSH}>25 \mathrm{mU} / \mathrm{L})\end{array}$} & \multirow{2}{*}{$\begin{array}{l}\text { Total } \\
\text { No. }\end{array}$} \\
\hline & No. & $\%$ & No. & $\%$ & \\
\hline Confirmed & $16^{\mathrm{a}}$ & 12.4 & 357 & 92.9 & 373 \\
\hline Transient & 49 & 38.0 & 11 & 2.9 & 60 \\
\hline False +ve & 57 & 44.2 & 9 & 2.3 & 66 \\
\hline Failure to recall & 7 & 5.4 & 7 & 1.9 & 14 \\
\hline Total +ve screening & 129 & 100.0 & 384 & 100.0 & 513 \\
\hline
\end{tabular}

a 9 cases were defaulters aged $>1$ month when screened. TSH = thyroid stimulating hormone.

term deliveries and 1 was a preterm delivery. The data indicated that the number of false positive recalls for preterm deliveries $(n=175)$ was greater than for the term group $(n=77)$. The total incidence of classical CAH detected through newborn screening in the UAE was 1:9030.

\section{Molecular DNA testing}

Of the 21 UAE national cases diagnosed as having $\mathrm{CAH} 18$ were confirmed genetically as CYP21 deficiencies, i.e. a mutation was found on both alleles: 10 were found to be homozygous for I2 splice mutation; 6 had homozygous CYP21A2 gene deletion; and 2 were heterozygous $I 2$ mutation. Three patients carried no CYP21 mutations by the methods used.

\section{Biotinidase deficiency}

Three confirmed cases of biotinidase deficiency by enzymatic testing ( 1 profound and 2 partial cases) were discovered during the pilot study for
25000 UAE national babies in 2010, an incidence of 1:8300.

\section{Molecular DNA testing}

Molecular testing was done for only 2 confirmed cases. The first patient had partial biotinidase deficiency and 2 heterozygous point mutations were detected [C186Y (Cys186Tyr) and D444H (Asp444His)]. The second patient had profound biotinidase deficiency and 4 mutations were detected [2 homozygous mutations F403V (Phe403Val) and 2 homozygous mutations $\mathrm{D} 444 \mathrm{H}$ (Asp444His)].

\section{Discussion}

Over the past 4 decades, many countries have developed screening programmes for newborns. An effective neonatal screening programme requires careful planning including education, administration, laboratory analysis, follow-up, management, evaluation and, most importantly, the commitment of all involved [6].

Our data indicated that the percentage uptake of neonatal screening in the UAE increased from 50\% in 1998 to reach $95 \%$ in 2010, with a rapid increase in 2003, although to levels still below the international coverage standard (99\%) [7]. This improvement could be due to increased community awareness, a better recall system for defaulters, perinatal health education being combined with a breastfeeding programme and the recording of the screening status of the baby on the child welfare record along with vaccination information. The rapid increase in the rate of uptake in 2003 can be attributed to starting cooperation with Dubai medical district.

The incidence of $\mathrm{CH}$ among the screened neonates was 1:1873. Of 373 confirmed cases, 357 had abnormal TSH level and 16 had persistent borderline TSH. Although high by international standards, the incidence of $\mathrm{CH}$ in the UAE was similar to that of other

\begin{tabular}{|c|c|c|c|c|c|}
\hline \multirow[t]{2}{*}{ Variable } & \multicolumn{2}{|c|}{$\begin{array}{c}\text { Borderline } \\
\text { (PHA 3-4 mg/dL) }\end{array}$} & \multicolumn{2}{|c|}{$\begin{array}{c}\text { Abnormal } \\
(\mathrm{PHA}>4 \mathrm{mg} / \mathrm{dL})\end{array}$} & \multirow{2}{*}{$\begin{array}{c}\text { Total } \\
\text { No. }\end{array}$} \\
\hline & No. & $\%$ & No. & $\%$ & \\
\hline PKU & - & 0.0 & 52 & 91.2 & $52^{\mathrm{a}}$ \\
\hline Benign hyperplasia & 11 & 15.1 & 5 & 8.8 & 16 \\
\hline Transient & 3 & 4.8 & - & 0.0 & 3 \\
\hline False +ve & 61 & 80.1 & - & 0.0 & 61 \\
\hline Total +ve screening & 75 & 100.0 & 57 & 100.0 & 132 \\
\hline
\end{tabular}

a51 cases were diagnosed as classical PKU and 1 case as PKU due to biopterin deficiency.

$P H A=$ phenylalanine . 


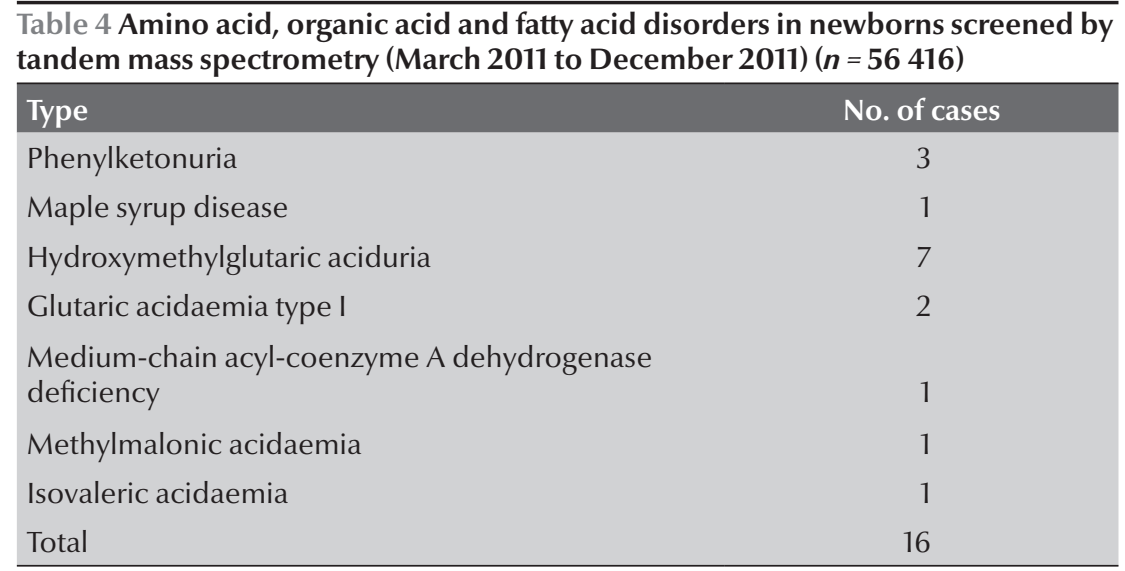

countries in the region such as Oman (1:2 200) and Saudi Arabia (1:2 666) [8].

In our research, which used $\mathrm{TSH}$ as an indicator for the evaluation of iodine deficiency, the high incidence of $\mathrm{CH}$ was unlikely to be explained through iodine deficiency mechanisms [9]. Of the 513 cases that screened positive, 60 transient cases were normal on follow up. This was explained by maternal history and investigations of autoimmune thyroid diseases in 14 cases and the presence of prematurity in 26 cases. However, in 20 cases no specific cause was found. This deserves further investigation, e.g. obtaining an accurate clinical history of perinatal exposure to iodine, maternal antithyroid medication intake during pregnancy, TSH receptor antibodies and thyroid peroxidase antibodies. These measures should help to decrease the recall rate when screening for $\mathrm{CH}$ and thus eliminate unnecessary stress to families, while contributing to a decline in the relatively high incidence of $\mathrm{CH}$ in the UAE [10].

There were 52 confirmed PKU cases over the 17-year period of screening (a relative incidence of 1:14 544); 51 were confirmed as classic PKU (phenylalanine $>20 \mathrm{mg} / \mathrm{dL}$, normal tyrosine and normal biopterin) and 1 case as PKU due to biopterin defect. The incidence of PKU in the UAE was similar to that of other Gulf countries [11]. The high incidence of false positives within the borderline group might be due to genetic heterogeneity of hyperphenylalaninaemia and might indicate a need to adjust the cut-off point for the screening test [12].
A total of 7 different mutations with no novel ones were detected by analysis of the PAH gene for 16 UAE national cases. Three splicing mutations were identified, with the following frequencies [IVS2+5G $>\mathrm{C}$ (37.5\%), IVS10-11G>A (12.5\%) and IVS9-2A>G (12.5\%)] and all were in the homozygous state. Four missense mutations were identified, with the following frequencies [R261Q (18.8\%), R252Q (6.3\%), P281L (6.3\%) and L48S (6.3\%)]. R261Q and R252Qwere in the homozygous state but P281L and L48S were in the compound heterozygous state. The different mutations also contribute to a difference in phenotype and response to pharmacological doses of tetrahydrobiopterin [13].

There were 16 cases of amino acid, organic acid and fatty acid disorders detected by MS/MS over a 10-month period in 2011 ( $n=56416$ neonates screed), an incidence 1:3 526. The majority ( 7 cases) were hydroxymethylglutaric aciduria.

The figures for SCD (0.04\%) and sickle-cell trait (0.83\%) were lower than earlier studies done in the UAE, which recorded an incidence of $1.9 \%$ for sickle-cell trait [14]. A study in Saudi Arabia reported an incidence range of 2\%-27\% for sickle-cell trait and about $1.4 \%$ for SCD with the highest rates in the Eastern region

\begin{tabular}{|c|c|c|c|c|c|c|}
\hline \multirow[t]{2}{*}{ Type } & \multicolumn{3}{|c|}{ Diseased } & \multicolumn{3}{|c|}{ Carriers } \\
\hline & No. identified & Incidence & Prevalence (\%) & No. identified & Incidence & Prevalence (\%) \\
\hline Sickle $\mathrm{Hb}$ & $231^{\mathrm{a}}$ & $1: 238$ & 0.04 & 4481 & 1:121 & 0.83 \\
\hline Non-sickle Hb & 80 & - & - & - & - & - \\
\hline $\mathrm{HbC}$ & 11 & - & - & 69 & - & 0.02 \\
\hline $\mathrm{HbD}$ & 65 & - & 0.01 & 592 & - & 0.11 \\
\hline $\mathrm{HbE}$ & 4 & - & - & 19 & - & - \\
\hline Unidentified band & - & - & - & $11^{\mathrm{b}}$ & & - \\
\hline Fetal $\mathrm{Hb}$ & - & - & - & $15^{c}$ & - & - \\
\hline
\end{tabular}

a Sickle-cell diseases: included 203 cases confirmed as homozygous SS and 28 cases confirmed as sickle/beta-thalassaemia; ${ }^{b} 3$ diagnosed as HbO Arab by family study; ' $\beta$-thalassaemia and hereditary persistence of fetal haemoglobin.

$\mathrm{Hb}=$ haemoglobin. 


\begin{tabular}{|c|c|c|c|}
\hline Variable & Term & Preterm & Total \\
\hline Screened population (no.) & 367436 & 11836 & 379272 \\
\hline Recalls (no.) & 717 & 337 & 1054 \\
\hline Recall rate $(\%)$ & 0.2 & 2.9 & 0.28 \\
\hline CAH confirmed (no.) & 41 & 1 & 42 \\
\hline False positive recalls (no.) & 77 & 175 & 252 \\
\hline
\end{tabular}

and the lowest in the Central region of the country [15]. Bahrain, on the other hand, had a rate of $11 \%-18 \%$ for sickle-cell trait, and an incidence of SCD of $2.1 \%$ among screened newborns [16]. Oman has reported an incidence of $10 \%$ for sickle-cell trait and about $0.4 \%$ for SCD [17].

The total incidence of classical $\mathrm{CAH}$ detected through newborn screening over a 10 -year period in the UAE was 1:9 030. This figure is in agreement with older data from UAE derived from clinically detected cases [Azzam A, unpublished data, 2000] and with the high incidence of CAH reported in Saudi Arabia [18]. The high incidence of CAH in UAE and Saudi Arabia can be explained by the high rate of consanguineous marriages. The considerable false-positive recall rate for neonatal $17 a$-hydroxyprogesterone screening causes a substantial economical burden and emotional stress for parents and makes genetic testing an important confirmatory tool for early and reliable $\mathrm{CAH}$ diagnosis. More than $90 \%$ of classical CAH are caused by mutations in or deletions of the CYP21A2 gene encoding steroid 21-hydroxylase [19]. Eighteen of 21 local cases diagnosed as having CAH were confirmed genetically as CYP21 deficiencies with 10 being homozygous for 12 splice mutation and 6 homozygous CYP21A2 gene deletion. Two cases had heterozygous I2 mutation and, as the 2 cases were confirmed biochemically, we propose that these cases may be attributable to compound heterozygosity of the CYP21 gene with a rare or de novo mutation. Three patients carried no CYP21 mutations by the method used and can also be attributable to other causes.

Three confirmed biotinidase deficiency cases by enzymatic testing ( 1 as profound and 2 as partial cases) were discovered during the pilot study for 25000 UAE national babies in 2010, an incidence of $1: 8300$. The disease incidence varies between countries, with higher incidences in countries with a high degree of consanguinity, such as Turkey, Saudi Arabia and Qatar (e.g. 1 in 12607 screened babies in Qatar) [20]. The estimated international incidence of combined partial and profound biotinidase deficiency is 60089 newborns (1:49 500 to 1:73 100) [21]. The first patient had partial biotinidase deficiency as confirmed by enzymatic and molecular testing. The molecular testing revealed 2 heterozygous mutations: C186Y mutations associated with profound biotinidase deficiency and $\mathrm{D} 444 \mathrm{H}$ mutations associated with partial biotinidase deficiency. The presence of these heterozygous mutations in the biotinidase gene were consistent with a diagnosis of partial biotinidase deficiency. The second patient had profound biotinidase deficiency as confirmed by enzymatic and molecular testing. The molecular testing revealed 4 mutations: 2 homozygous F403V pathogenic mutations and 2 homozygous $\mathrm{D} 444 \mathrm{H}$ mutations consistent with partial biotinidase deficiency. The presence of 2 double homozygous mutations in the biotinidase gene was consistent with a diagnosis of profound biotinidase deficiency. No previous reports of similar cases were found in literature for the patient having 2 homozygous alterations (F403V; $\mathrm{D} 444 \mathrm{H}$ ). These alterations could be considered as novel mutations and could be related to a deletion of the exon, parental consanguinity, uniparental disomy or by chance alone. One way to address this issue is to test the parents and determine if they are carriers of the above mentioned alterations [22].

\section{Conclusions}

Based on the statistical data of the incidence of screened diseases in the UAE and the success achieved in early detection, treatment and follow-up, we emphasize the importance of expanding the programme capacity.

\section{Acknowledgements}

We would like to thank and express our gratitude to the laboratory staff, colleagues at the Central Department of Maternal and Child Health, districts coordinators and physicians across UAE and finally to all those who shared by their time and effort in the progress of the national neonatal screening programme.

Funding: the study was funded was by the Ministry of Health, UAE.

Competing interests: None declared. 


\section{References}

1. American Academy of Pediatrics. Committee on Genetics: issues in newborn screening. Pediatrics, 1992, 89:345-349.

2. Chace DH, Kalas TA, Naylor EW. Use of tandem mass spectrometry for multianalyte screening of dried blood specimens from newborns. Clinical Chemistry, 2003, 49:1797-1817.

3. Song F et al. Phenylketonuria mutations in Northern China. Molecular Genetics and Metabolism, 2005, 86:S107-S118.

4. Krone $\mathrm{N}$ et al. Multiplex minisequencing of the 21-hydroxylase gene as a rapid strategy to confirm congenital adrenal hyperplasia. Clinical Chemistry, 2002, 48:818-825.

5. Hymes J, Stanley CM, Wolf B. Mutations in BTD causing biotinidase deficiency. Human Mutation, 2001, 18:375-381

6. Loeber G, Webster D, Aznarez A. Quality evaluation of newborn screening programs. Acta Paediatrica, Supplement, 1999, 88:3-6.

7. American Academy of Pediatrics. Update of newborn screening and therapy for congenital hypothyroidism. Pediatrics, 2006, 117:2290-2303.

8. Al-Nuaim A et al. Neonatal screening for congenital hypothyroidism. Incidence, imaging, feasibility and difficulties of a nationwide programme. Annals of Saudi Medicine, 1992, 12:129-134.

9. Al-Hosani $\mathrm{H}$ et al. Prevalence of iodine deficiency disorders in the United Arab Emirates measured by raised TSH levels. Eastern Mediterranean Health Journal, 2003, 9:123-130

10. Abu-Osba YK et al. Comprehensive newborn screening programmes: ARAMCO experience, the national need and recommendations. Annals of Saudi Medicine, 1992, 12:235-240.

11. Holtzman NA, Meek AG, Mellits ED. Neonatal screening for phenylketonuria. I. Effectiveness. Journal of the American Medical Association, 1974, 229:667-670.

12. Veale AMO. Screening for hereditary metabolic disorders. Screening for phenylketonuria. In: Bickel H, Guthrie R, Hammersen G, eds. Neonatal screening for inborn errors of metabolism. Berlin, Springer-Verlag, 1980:7-18.
13. Harvey L, Burton B, Cederbaum S. Recommendations for evaluation of responsiveness to tetrahydrobiopterin $\mathrm{BH} 4$ in phenylketonuria and its use in treatment. Molecular Genetics and Metabolism, 2007, 92:287-291.

14. White JM et al. Red cell genetic abnormalities in Peninsular Arabs: sickle haemoglobin, G6PD deficiency, and alpha and beta thalassaemia. Journal of Medical Genetics, 1986, 23:245-251.

15. Nasserullah Z et al. Neonatal screening for sickle-cell disease, glucose-6-phosphate dehydrogenase deficiency and alpha thalassemia in Qatif and Al Hasa. Annals of Saudi Medicine, 1998, 18:289-292.

16. Mohammed AM et al. Haemoglobinopathies and glucose6-phosphate dehydrogenase deficiency in hospital births in Bahrain. Annals of Saudi Medicine, 1992, 12:536-539

17. Rajab A. Prevention of genetic disorders in Oman. Ambassadors, 1998, 1:5 [online magazine] (http://ambassadors.net/ archives/issue3/select-oman.htm, accessed 26 August 2013).

18. El-Hamzi M et al. Consanguinity among the Saudi Arabian population. American Journal of Medical Genetics, 1995, 32:623-626

19. Kösel $\mathrm{S}$ et al. Rapid second-tier molecular genetic analysis for congenital adrenal hyperplasia attributable to steroid 21-hydroxylase deficiency. Clinical Chemistry, 2005, 51:298-304.

20. Lindner $M$ et al. Implementation of extended neonatal screening and a metabolic unit in the State of Qatar: developing and optimizing strategies in cooperation with the Neonatal Screening Center in Heidelberg. Journal of Inherited Metabolic Disease, 2007, 30:522-529.

21. Wolf B. Worldwide survey of neonatal screening for biotinidase deficiency. Journal of Inherited Metabolic Disease, 1991, 14:923-927.

22. Cowan TM, Blitzer MG, Wolf B. Technical standards and guidelines for the diagnosis of biotinidase deficiency. Genetics in Medicine, 2010, 12:464-470. 